\title{
Trabectedin for inoperable or recurrent soft tissue sarcoma in adult patients: a retrospective cohort study
}

\author{
Fernando A. Angarita' ${ }^{1}$ Amanda J. Cannell ${ }^{1}$, Albiruni R. Abdul Razak ${ }^{2,3,4}$, Brendan C. Dickson 5,6 \\ and Martin E. Blackstein $2,3,4^{*}$
}

\begin{abstract}
Background: Trabectedin is an antineoplastic agent used for patients with soft tissue sarcoma (STS) who fail standard-of-care treatment. Real-world data of its performance is scarce. This study evaluates the safety and effectiveness of trabectedin for patients with advanced STS who were treated at a high-volume sarcoma center.

Methods: A retrospective chart review was performed on 77 patients treated with trabectedin ( $24 \mathrm{~h}$ infusion q3w) between 01/2005 and 05/2014. Data regarding safety, objective radiological response, progression-free and overall survival were analyzed.

Results: Median age at treatment onset was 52y [interquartile range (IQR): 45-61y]. Tumors included leiomyosarcoma $(41.6 \%)$, liposarcoma (18.2\%), and synovial sarcoma (13\%). Trabectedin was provided as $\geq$ third-line chemotherapy in $71.4 \%$. Median number of cycles was 2 (range: 1-17). Dose reduction and treatment delays occurred in 19.5 and $40.3 \%$, respectively. Toxicities occurred in $78 \%$, primarily for neutropenia or elevated liver enzymes. Two patients died secondary to trabectedin-induced rhabdomyolysis. Treatment was discontinued because of disease progression (84.7\%), toxicity (10 \%), and patient preference (5\%). Partial response or stable disease occurred in 14.1 and $33.8 \%$, respectively, while $52.1 \%$ developed progressive disease. Median progression-free survival was $1.3 \mathrm{~m}$ (IQR: 0.7-3.5 m) and was significantly higher in patients lacking severe toxicities or progressive disease. Median overall survival was $6.7 \mathrm{~m}$ (IQR: $2.3-12.7 \mathrm{~m}$ ) and was significantly higher in patients with leiomyosarcoma or liposarcoma relative to other histologies.
\end{abstract}

Conclusions: Trabectedin has an acceptable safety profile as an anti-tumor agent. Our data further suggest there may be some benefit in using trabectedin particularly in patients with leiomyo- or liposarcoma who failed standard-of-care agents.

\section{Background}

Soft tissue sarcomas (STS) are rare solid cancers of mesenchymal cell origin accounting for $<1 \%$ of adult cancers [1]. Considerable heterogeneity exists with over 50 histologic types of STS, each with distinct clinical behaviour. Despite this heterogeneity, adult patients

\footnotetext{
* Correspondence: martin.blackstein@utoronto.ca

This work was previously presented at the Connective Tissue Oncology Society (CTOS) 20th Annual meeting held in Berlin, Germany on October 15 - 18, 2014.

2Department of Medical Oncology, Mount Sinai Hospital, Toronto, ON, Canada

${ }^{3}$ Department of Medical Oncology, Princess Margaret Cancer Centre, University Health Network, Toronto, ON, Canada

Full list of author information is available at the end of the article
}

with advanced STS are generally treated similarly with palliative-intent chemotherapy. Few agents have known anti-tumor activity in advanced STS, but generally provide limited benefits in survival outcome. Guidelines recommend anthracycline-based chemotherapy as firstline treatment for most advanced STS [2, 3] with a $26 \%$ response rate [4]. Another drug used as first-line treatment is ifosfamide, which with or without doxorubicin offers a response rate of $\sim 25 \%$ [5]. Treatment options for patients who fail first-line treatment include gemcitabine/docetaxel [3], but additional agents are scarce.

Trabectedin is the synthetic version of an anti-cancer alkaloid agent originally isolated from the Caribbean sea squirt Ecteinascidia turbinate [6]. Trabectedin covalently 
binds to the DNA minor groove at guanine nucleotides of specific sequences to inhibit gene activation and repair mechanisms and induce lethal DNA double-strand breaks that ultimately lead to cell cycle arrest [7]. Additionally, recent studies suggest pleiotropic properties [8-11]. Trabectedin selectively targets macrophages and down-regulates the production of pro-inflammatory mediators, changing the tumor microenvironment and contributing to anti-cancer activity [8-10]. Trabectedin also promotes cancer cell differentiation, specifically in myxoid liposarcoma by modulating the transcription of genes crucial for adipocytic differentiation [11].

Trabectedin has shown efficacy as salvage chemotherapy in patients with advanced STS in three phase II trials [12-14], chemotherapy-naive patients with unresectable advanced disease [15], and in compassionate use programs [16-18]. An open-label, randomized, phase II study evaluated two regimens in patients with unresectable advanced or metastatic liposarcoma or leiomyosarcoma [19]. This study established that trabectedin $\left(1.5 \mathrm{mg} / \mathrm{m}^{2}\right.$ given as a 24-h intravenous infusion $\mathrm{q} 3 \mathrm{w}$ ) provided significantly better disease control over weekly $0.58 \mathrm{mg} / \mathrm{m}^{2}$ by improving time to progression (TTP) and progression-free survival (PFS). In 2007, based on these results, trabectedin was approved in several countries for use in STS patients who fail standard treatments or are unsuited to receive first-line agents [20, 21].

The effectiveness and safety profile of trabectedin in the aforementioned studies may differ from that of real clinical settings as patients typically go through rigorous enrolment processes before entering clinical trials. Institutional case series provide a suitable means to obtain real-world data. Therefore, our study aimed to evaluate the safety and effectiveness of trabectedin in patients with inoperable or recurrent STS treated at a highvolume academic sarcoma center in North America.

\section{Methods \\ Study design}

Research ethics board approval at Mount Sinai Hospital (MSH), Toronto, ON, Canada was obtained in order to identify patients treated with trabectedin from the University of Toronto Sarcoma Group's medical oncology and pharmacology database. Informed consent was obtained in order to include patients into the database. A retrospective chart review was performed from medical records of patients who initiated treatment between 01/01/2005 and $05 / 30 / 2014$. Inclusion criteria included $\geq 18$ years old (y); histologically confirmed STS; patients with locally advanced, metastatic, inoperable, recurrent or disease progression after first-line treatment; and at least one treatment cycle. Patients with gastrointestinal stromal tumors were excluded.

\section{Data collection}

Data was extracted by one author (FAA) and $10 \%$ of data was independently corroborated by two additional authors (AJC and MEB). Extracted data included patient demographics and medical history, STS details, pretrabectedin treatment information, trabectedin information, post-trabectedin treatment information and follow-up information.

\section{Clinical practice}

The University of Toronto Sarcoma Group treats patients at both Mount Sinai Hospital and Princess Margaret Cancer Centre (PM), two high-volume adult sarcoma centres for the province of Ontario. Tumor specimens were classified according to the World Health Organization (WHO) system by an expert soft tissue pathologist (BCD). Patients are generally referred from regional health centres for multidisciplinary management.

To be eligible for treatment patients had to meet the following criteria: $\geq 18 y$, biopsy-proven STS, documented unresectable advanced or metastatic tumor, either failure or intolerance to doxorubicin and/or ifosfamide, currently not receiving anti-cancer treatment, Eastern Cooperative Oncology Group (ECOG) performance status $\leq 1$; adequate bone marrow reserve (neutrophils $>1500 / \mathrm{mm}^{3}$ and platelets $>100,000 / \mathrm{mm}^{3}$ ); adequate renal function (serum creatinine $<120 \mu \mathrm{mol} / \mathrm{L}$ or calculated creatinine clearance by Cockroft method $>60 \mathrm{~mL} / \mathrm{min}$ ); and adequate hepatic function (bilirubin $>30 \mu \mathrm{mol} / \mathrm{L}$, aspartate aminotransferase (AST) and alanine transaminase (ALT) $\mathrm{T}<1.5 \mathrm{U} /$ $\mathrm{L}$ or $<2.5 \mathrm{U} / \mathrm{L}$ if liver metastases, alkaline phosphatase (ALP) $<2.5 \mathrm{U} / \mathrm{L}$ and albumin $>25 \mathrm{~g} / \mathrm{L}$ ). Contraindications included known history of hypersensitivity to trabectedin or its components, active serious and/or uncontrolled infection, left ventricular ejection fraction below lower normal limit, concomitant live vaccines, creatine kinase $(\mathrm{CK})>2.5 \mathrm{x}$ upper normal limit, elevated bilirubin and breast feeding.

Trabectedin was generally given at the recommended starting dose $\left(1.5 \mathrm{mg} / \mathrm{m}^{2}\right)$ as a 24 -h continuous intravenous infusion $\mathrm{q} 3 \mathrm{w}$. Trabectedin was administered via a portable infusion pump that enabled outpatient treatment. Before each cycle patients were assessed to confirm adequate renal, hepatic and bone marrow reserve function as well as overall performance status. Anti-emetic prophylaxis with corticosteroids (20 mg of dexamethasone intravenously administered 30 min pre-trabectedin) was provided. Dose reductions ( $20 \%$ intervals) were made in the event of toxicities occurring during the previous cycle. Once the toxicity resolved the dose was readjusted at the discretion of the medical oncologist. Dose adaptations were similar to those applied in previously published protocols [12, 14]. There were no pre-defined limits to the number of cycles therefore patients with non-progressive disease and no adverse 
events continued receiving treatment until progression, grade 4 toxicities, and/or patient preference. Response to treatment was assessed every two cycles by CT scans, which were reviewed by the treating medical oncologist.

\section{Classifications}

Toxicity was retrospectively assessed using the Common Terminology Criteria for Adverse Events (CTCAE) v4.03 classification [22]. Because of the study's retrospective nature, only hematological and biochemical results could be assessed by toxicity scale while clinical adverse events were only described. Best response to treatment was determined by two authors (FAA and MEB) who retrospectively reviewed CT scans and used Response Evaluation Criteria in Solid Tumors (RECIST) v.1.1 to categorize the response as either complete remission (CR), partial remission (PR), stable disease (SD) or progressive disease (PD) [23].

\section{Statistical analysis}

Statistical analyses were performed using SPSS 20 (IBM, Armonk, NY, USA). Data were expressed as median with the interquartile range (IQR) and percentage, unless otherwise specified. Survival analyses were conducted by Kaplan-Meier method and compared with log-rank test. PFS was calculated from the date of first dose of trabectedin to the date of disease progression as documented on CT scan. Patients who only received one cycle of trabectedin or died before their first on-treatment CT scan were excluded from PFS analysis. Patients were censored at time of death or last follow-up at MSH/PM, whichever occurred first. Overall survival (OS) was calculated from the date of first dose of trabectedin to the date of death or last follow-up at MSH/PM, whichever occurred first. The cut-off date for follow-up in this study was March 31,2015 . Statistical significance was set at $p$-value $<0.05$.

\section{Results}

\section{Patient and tumor characteristics}

A total of 77 patients were treated with trabectedin for unresectable advanced or metastatic STS (Table 1). Patients had a median age of 52y (IQR: 45-61y) and were predominately female (62.3\%). Overall patients had a good performance status before treatment (97.4\%). The majority of patients $(57.1 \%)$ had at least one comorbidity of which hypertension $(n=13)$, hypothyroidism $(n=7)$, diabetes mellitus $(n=6)$, depression $(n=6)$, and smoking $(n=6)$ were the most common. Nine patients $(11.7 \%)$ had a prior history of cancer including bladder $(n=3)$, breast $(n=2)$, thyroid $(n=2)$, and lymphoma $(n=2)$.

The most common STSs included leiomyosarcoma (41.6\%), liposarcoma (18.2\%), and synovial sarcoma (13\%). Approximately $27 \%$ of patients had a variety of rare histologies ("other sarcoma"), which included spindle cell sarcoma, fibrosarcoma, clear cell sarcoma,
Table 1 Patient and tumour characteristics at time of starting trabectedin

\begin{tabular}{|c|c|}
\hline Variable & N (\%) \\
\hline Age (years), median (IQR) & $52(45-61)$ \\
\hline \multicolumn{2}{|l|}{ Gender } \\
\hline Male & $29(37.7)$ \\
\hline Female & $48(62.3)$ \\
\hline \multicolumn{2}{|l|}{ ECOG performance status } \\
\hline 0 & $32(41.6)$ \\
\hline 1 & $43(55.8)$ \\
\hline 2 & $2(2.6)$ \\
\hline \multicolumn{2}{|l|}{ Number of comorbidities } \\
\hline 0 & $33(42.9)$ \\
\hline 1 & $19(24.7)$ \\
\hline$\geq 2$ & $25(32.4)$ \\
\hline \multicolumn{2}{|l|}{ Prior history of cancer } \\
\hline No & $68(88.3)$ \\
\hline Yes & $9(11.7)$ \\
\hline \multicolumn{2}{|l|}{ Histology } \\
\hline Leiomyosarcoma & $32(41.6)$ \\
\hline Others & $21(27.2)$ \\
\hline Liposarcoma & $14(18.2)$ \\
\hline Synovial sarcoma & $10(13)$ \\
\hline \multicolumn{2}{|l|}{ Grade } \\
\hline I & $8(10.4)$ \\
\hline$\|$ & $16(20.8)$ \\
\hline III & $27(35.1)$ \\
\hline Not graded/recorded & $28(36.4)$ \\
\hline \multicolumn{2}{|l|}{ Site of primary tumour } \\
\hline Thorax & $6(7.8)$ \\
\hline Abdomen/pelvis & $11(14.3)$ \\
\hline Retroperitoneum & $13(16.9)$ \\
\hline Uterus & $21(27.3)$ \\
\hline Extremity & $26(33.8)$ \\
\hline \multicolumn{2}{|c|}{ Site of local recurrence $(n=20)$} \\
\hline Thorax & $4(20)$ \\
\hline Abdomen/pelvis & $12(50)$ \\
\hline Retroperitoneum & $3(15)$ \\
\hline Extremity & $1(5)$ \\
\hline \multicolumn{2}{|l|}{ Site of metastasis $(n=57)$} \\
\hline Brain & $1(1.8)$ \\
\hline Lung & 46 (80.7) \\
\hline Thorax & $4(7)$ \\
\hline Liver & $15(26.3)$ \\
\hline Abdomen/pelvis & $13(22.8)$ \\
\hline Bone & $14(24.6)$ \\
\hline
\end{tabular}


Table 1 Patient and tumour characteristics at time of starting trabectedin (Continued)

\begin{tabular}{ll}
\hline Extent of tumour & \\
Inoperable primary tumour & $13(16.9)$ \\
Locally recurrent & $13(16.9)$ \\
Recurrent metastatic tumour & $44(57.1)$ \\
Locally recurrent and metastasis & $7(9.1)$ \\
\hline ECOG Eastern Cooperative Oncology Group
\end{tabular}

high-grade pleomorphic undifferentiated sarcoma and rhabdomyosarcoma. Of patients who had information available, the majority of tumors were high grade (55.1\%).

Tumors were primarily localized in in the torso (66.2\%). The most common sites of primary disease were uterus (27.3\%), retroperitoneum (16.9\%), and abdomen/pelvis (14.3\%). At the time of starting trabectedin 51 (66.2 \%) patients had one or more metastasis. Patients had metastasis with the following number of sites involved: one (52.6\%), two $(33.3 \%)$, and three or more (14\%). Anatomical distribution of metastasis was as follows: lung (80.7\%), liver (26.3\%), abdomen/pelvis (22.8 \%), and bone (24.6\%)

\section{Treatment before trabectedin}

All 77 patients received first-line chemotherapy before staring trabectedin. The majority of patients $(71.4 \%)$ received at least two lines of chemotherapy before starting trabectedin. Prior to trabectedin, 11 patients underwent radiation therapy with the following intent: neoadjuvant (9.1\%), adjuvant (36.7\%), and palliative (54.5\%). A total of $64(83.1 \%)$ patients had surgery for their primary tumor prior to starting trabectedin while the remaining 13 patients had inoperable tumors. Margin status after surgery for patients' primary STS was as follows: R0 (76.6 \%), R1 (20.3 \%), and R2 (3.1\%). Local recurrence was diagnosed in $20(31.3 \%)$ patients. Distribution of site of local recurrence was as follows: abdomen/pelvis (50\%), thorax (20\%), retroperitoneum (15\%), and extremity (5\%). Twenty-three patients underwent additional surgery including positive margin excision (17.4\%), local recurrence excision (17.4\%), and metastectomy (65.2\%). Trabectedin was provided for patients with recurrent metastatic (57.1\%), locally recurrent (16.9\%), inoperable primary $(16.9 \%)$, and both locally recurrent and metastatic $(9.1 \%)$ tumours.

\section{Trabectedin treatment characteristics}

Median time from diagnosis to start of trabectedin was 22.4 months $(\mathrm{m})$ (IQR: 13.3-44.9 m). Median number of cycles of trabectedin was 2 (range: 1-17) during a median time of $1.5 \mathrm{~m}$ (range: $0.3-16 \mathrm{~m}$ ). Trabectedin was primarily provided as second- and third-line chemotherapy in $28.6 \%$ and $44.2 \%$ patients, respectively; while the remaining $27.3 \%$ received it as $\geq 4$-line treatment. Of the 77 patients, 2 patients were started on trabectedin after developing severe toxicities with other lines of chemotherapy while the remaining 75 patients received treatment due to disease progression.

The majority of patients $(96.1 \%)$ started treatment at a dose of $1.5 \mathrm{mg} / \mathrm{m}^{2}$. Three patients started treatment at $1.2 \mathrm{mg} / \mathrm{m}^{2}$ because they were considered frail; two of these patients eventually had their dose increased to $1.5 \mathrm{mg} / \mathrm{m}^{2}$ because they tolerated treatment. Frequency and reasons for dosage and schedule modifications are depicted in Fig. 1. A total of 15 patients (19.5 \%) required dose reductions primarily owing to low absolute neutrophil count (ANC) (40\%), followed by hepatotoxicity $(26.7 \%)$ and clinical reasons (20\%) (Fig. 1a). In the majority of cases, patients required a single dose reduction $(86.7 \%)$, but dosage was generally readjusted to normal (66.7 \%). A total of 31 (40.3\%) patients had treatment delays primarily because of low ANC (61.3\%), catheter problems (12.9\%), and personal reasons (12.9 \%) (Fig. 1b). Number of treatment delays per patient was as follows: one (80.6\%), two (16.1\%), and three $(3.2 \%)$. Trabectedin therapy was discontinued in 72 patients (93.5 \%) because of disease progression (84.7\%), severe adverse events (9.7\%), and patient decision $(5.6 \%)$ (Fig. 1c). Currently five patients are undergoing treatment with trabectedin.

\section{Trabectedin-related toxicities}

A total of 150 hematological and/or biochemical toxicities occurred in 60 patients (Table 2). The median number of hematological and/or biochemical toxicities per patient was 2 (IQR: 1-3). The most common toxicities included low ANC (29.3\%) and elevated liver enzymes $(26 \%)$. Events of severe toxicity (CTCAE grade $\geq 3$ ), primarily occurred because of elevation of liver enzymes (18.7 \%), low ANC (12.7 \%), and elevated CK (3.3 \%). A total of 25 clinical adverse events occurred with the following distribution: nausea/vomiting $(n=18)$, fatigue $(n=5)$, diarrhea $(n=1)$, and leg edema $(n=1)$.

Deaths attributed to drug-related events were reported in two patients both of which were due to rhabdomyolysis. One patient with recurrent metastatic poorly differentiated leiomyosarcoma in the abdomen developed elevated CK after two cycles. The patient was admitted for rhabdomyolysis and treated, but died from acute tubular necrosis. Another patient with an inoperable retroperitoneal grade III malignant fibrous histiocytoma died after three cycles of trabectedin. The patient presented with severe bilateral lower limb pain and edema and blood work suggested ongoing rhabdomyolysis and acute renal failure. Despite treatment, the CK continued to increase, reaching 18,400U/L, until the patient eventually developed anuria and died secondary to acute renal failure. 


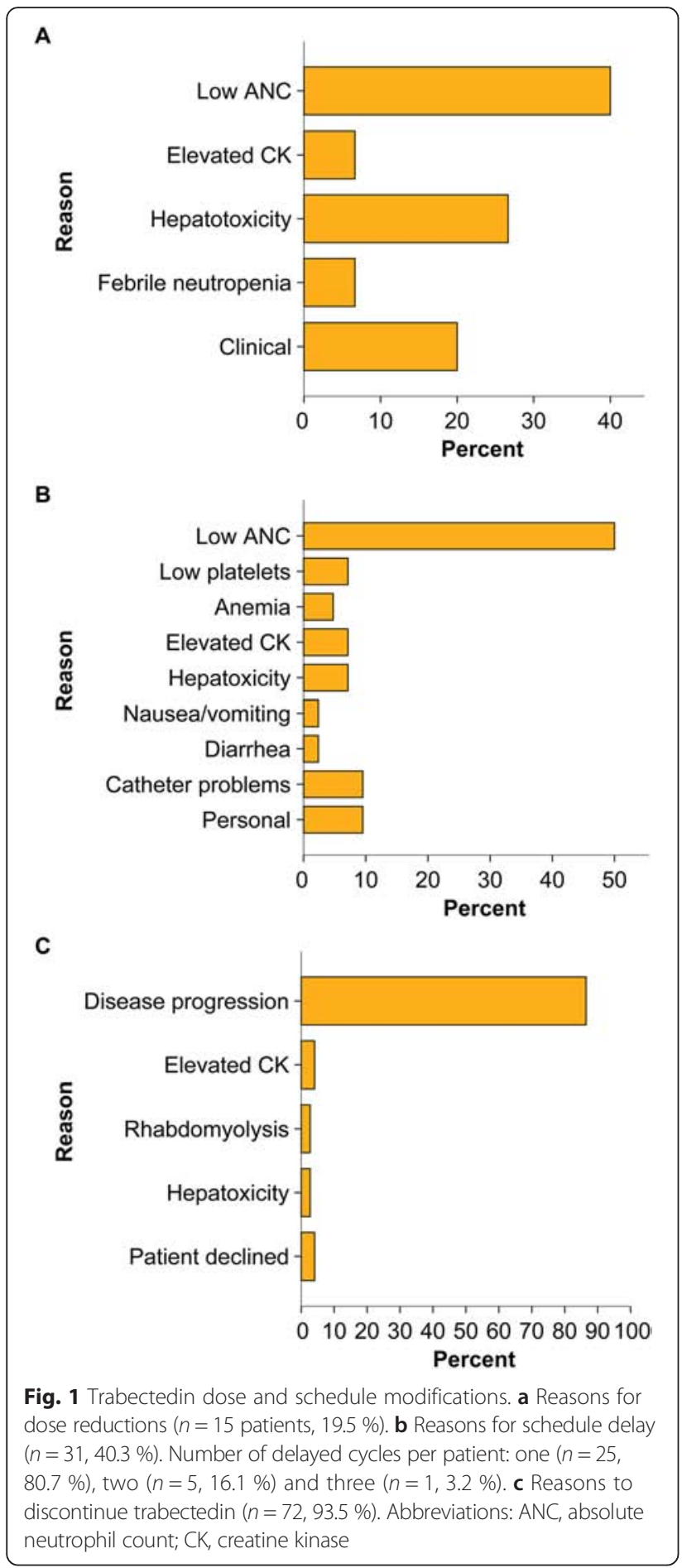

\section{Effectiveness}

Seventy-one were assessed for effectiveness. Six patients were excluded because they did not have on-treatment CT scans at the study cut-off date: three patients stopped treatment after cycle 1 for personal reasons, one patient had only one cycle, and two patients had early, severe toxicities requiring treatment suspension.
Table 2 Trabectedin-related toxicities

\begin{tabular}{llllll}
\hline Type of toxicity & $\begin{array}{l}\text { Grade } 1 \\
\mathrm{n}(\%)\end{array}$ & $\begin{array}{l}\text { Grade } 2 \\
\mathrm{n}(\%)\end{array}$ & $\begin{array}{l}\text { Grade } 3(\%) \\
\mathrm{n}(\%)\end{array}$ & $\begin{array}{l}\text { Grade } 4 \\
\mathrm{n}(\%)\end{array}$ & $\begin{array}{l}\text { Grade } 5 \\
\mathrm{n}(\%)\end{array}$ \\
\hline Hematological & & & & & \\
$\quad$ Anemia & $8(10.4)$ & $3(3.9)$ & $6(7.8)$ & - & - \\
Low ANC & $1(1.3)$ & $24(32.4)$ & $15(20.3)$ & $4(5.2)$ & - \\
& $5(6.5)$ & - & $1(1.3)$ & $2(2.6)$ & - \\
Thrombocytopenia & & & & & \\
Lymphoenia & $1(1.3)$ & - & $1(1.3)$ & - & - \\
Febrile & - & - & $2(2.6)$ & - & - \\
neutropenia & & & & & \\
Biochemical & & & & & \\
Elevated ALP & $12(15.6)$ & $2(2.6)$ & $4(5.2)$ & $2(2.6)$ & - \\
Elevated GGT & $10(12.9)$ & $2(2.6)$ & $5(6.5)$ & $2(2.6)$ & - \\
Elevated CK & $4(5.2)$ & $1(1.3)$ & $1(1.3)$ & $2(2.6)$ & $2(2.6)$ \\
Elevated AST & $3(3.9)$ & $2(2.6)$ & $5(6.5)$ & $2(2.6)$ & - \\
Elevated ALT & $6(7.8)$ & $1(1.3)$ & $5(6.5)$ & $3(3.9)$ & - \\
Elevated bilirubin & - & - & $1(1.3)$ & - & -
\end{tabular}

$A L P$ alkaline phosphatase, $A L T$ alanine transaminase, $A N C$ absolute neutrophil count, AST aspartate aminotransferase, $C K$ creatine kinase, GGT

gamma-glutamyl transferase

Figure 2 illustrates best response to trabectedin as measured by RECIST. While CR was not observed in any patient, partial PR and SD were recorded as best response in $10(14.1 \%)$ and $24(33.8 \%)$ patients, respectively. The remaining 37 (52.1\%) patients showed PD. Figure 3 depicts the distribution of best type of response to trabectedin according to tumor histology. Trabectedin tended to induce PR in patients with liposarcoma $(21.4 \%)$ and leiomyosarcoma (12.5\%) (Fig. 4).

The median PFS was $1.3 \mathrm{~m}$ (IQR: 0.7-3.5 m). Fig. 5a-e depicts PFS stratified by factors with potential impact on outcome. PFS was significantly higher in patients who had grade $<3$ toxicities relative to those with grade $\geq 3$ toxicities ( $1 \mathrm{~m}$ versus $2 \mathrm{~m}, p=0.02)$. PFS was also significantly higher in patients who had $\mathrm{PR}$ or $\mathrm{SD}$ relative to those with PD (PR: $5 \mathrm{~m}$ versus PD: $1 \mathrm{~m}, p<0.0001$ and SD: $2 \mathrm{~m}$ versus PD: $1 \mathrm{~m}, p<0.0001)$. Trabectedin did not induce significant improvements in PFS depending on histology, extent of tumor at presentation, or current number of line of chemotherapy. The median follow-up time was $6.6 \mathrm{~m}$ (IQR: $2.3-12.7 \mathrm{~m}$ ). The median OS for this cohort was $6.7 \mathrm{~m}$ (IQR: 2.3-12.7 m). Fig. $5 \mathrm{f}-\mathrm{j}$ depicts OS stratified by factors with potential impact on outcome. Patients with leiomyosarcoma or liposarcoma had significantly higher OS relative to other types (leioymyosarcoma: $12.2 \mathrm{~m}$ versus others: $3.7 \mathrm{~m}, p<0.0001$ and liposarcoma: $10.5 \mathrm{~m}$ versus others: $3.7 \mathrm{~m}, p=0.002)$. OS was significantly higher in patients who had PR relative to those with PD (PR: $16 \mathrm{~m}$ versus PD: $6 \mathrm{~m}, p=0.003$ ). OS did not improve depending on extent of tumor at presentation, current number of line of chemotherapy, 


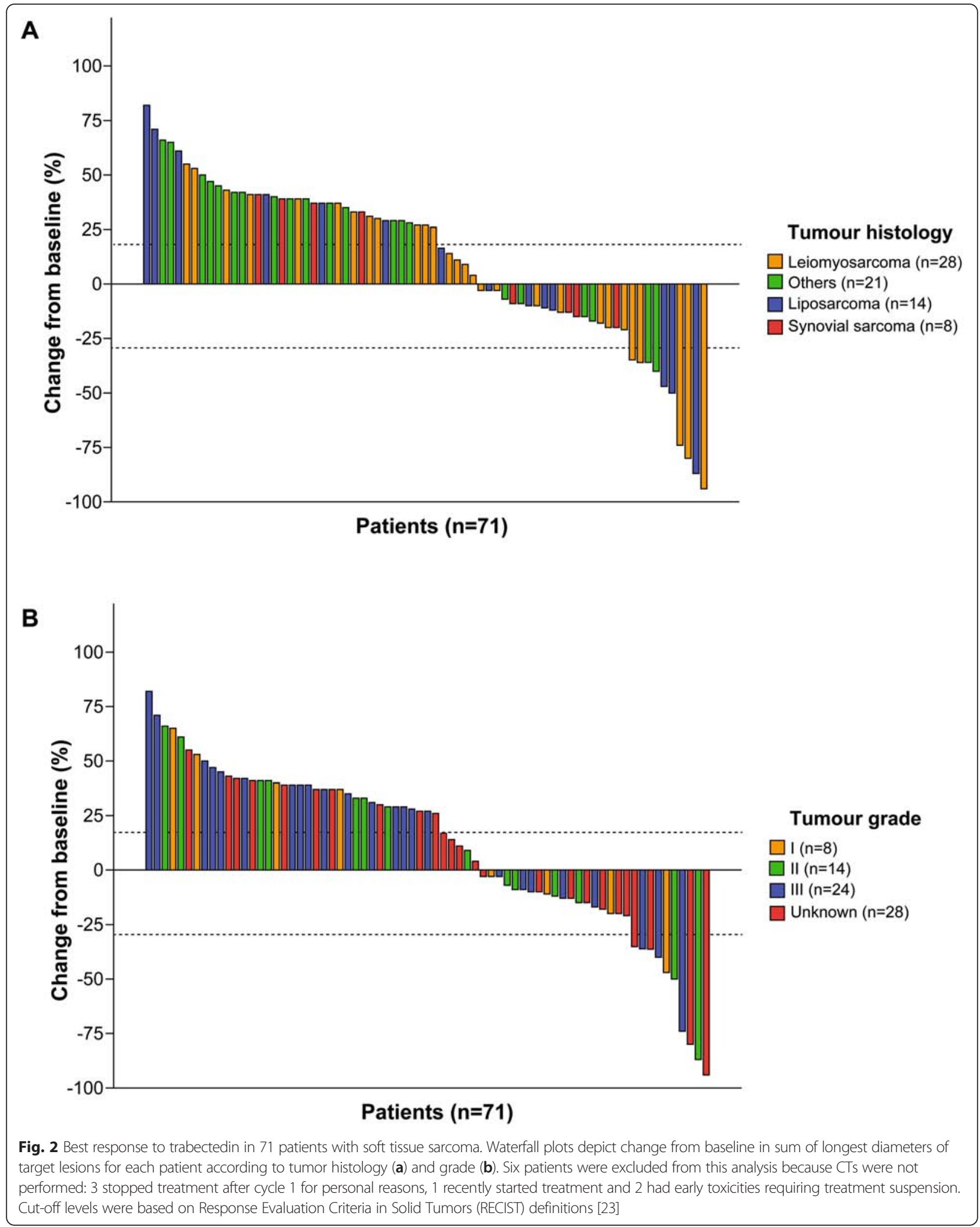




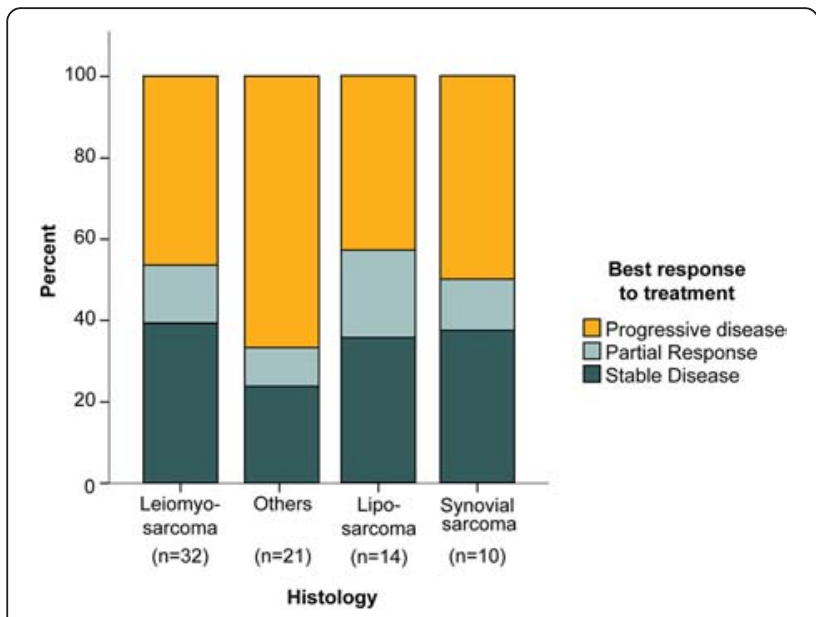

Fig. 3 Distribution of best type of response to trabectedin according to tumor histology. Best response was assessed using Response Evaluation Criteria in Solid Tumors (RECIST) $(n=71)$

or severity of toxicity. At the end of the follow-up period, $54(70.1 \%)$ patients had died of their disease $(n=52)$ or trabectedin-related causes $(n=2)$. Twentythree patients are alive and undergoing the following treatments: other chemotherapies $(n=9)$, palliative care $(n=6)$, trabectedin $(n=5)$, and targeted therapies $(n=3)$.

\section{Discussion}

This study assessed the safety and effectiveness of trabectedin in a 'real-world' setting in patients with advanced STS. Between 2005 and 2014, our high-volume adult sarcoma centre treated 77 patients with trabectedin, the largest retrospectively published cohort of patients in North America to date. Our cohort resembles that of other larger studies in terms of patient and tumor characteristics [13, 24, 25]. Our results confirm that trabectedin is a well-tolerated agent that appears to induce some response in patients with advanced STS who previously failed standard-of-care chemotherapy. Trabectedin has a manageable safety profile with common toxicities including reversible low ANC, anemia, thrombocytopenia and hepatotoxicity and rare, severe clinical consequences such as elevated CK and rhabdomyolysis. Cumulative toxicities were not observed. At best, trabectedin induced a PR, particularly in patients with lipo- and leiomyosarcoma. Patients who had grade $\geq 3$ toxicities and PD had significantly worse PFS relative to their counterparts. OS was significantly higher in patients with leiomyo- or liposarcoma relative to their counterparts. Given that our treatment inclusion criteria were less restrictive than those of clinical studies, our study depicts how trabectedin acts in a real clinical setting.

Trabectedin treatment in our cohort was reasonably well tolerated with an overall safety profile consistent with that of previous studies. As previously reported, the most common toxicities included self-limiting low ANC and elevated liver enzymes $[25,26]$. Neutropenia was the most common drug-related toxicity and its incidence and severity is particularly higher with the dosing schedule used by our group [25]. Neutropenia followed a predictable and reversible course and was rarely associated with fever $(1.9 \%)$ or infection $(1.8 \%)$ as also noted in our cohort [25, 27]. Elevated liver enzymes occurred mainly in the first weeks of the first cycle and levels generally returned to baseline by day one of cycle two [25, 28]. Excluding patients with a known history of active liver disease and closely monitoring patients provides an adequate opportunity to adjust treatment. Notably, the incidence of bilirubin and alkaline phosphatase elevations was low and was not cumulative as previously reported [25].

In this study, the prevalence of grade $\geq 3$ elevated liver enzymes and low ANC was within the rates reported in previous studies (low ANC: $33-61 \%$ and transaminitis: $20-57 \%$ ) $[12-15,29,30]$. Despite the frequency of grade $\geq 3$ toxicities, they only accounted for $2.8 \%$ of the reasons why trabectedin was discontinued. Additionally events requiring in-hospital management affected $10 \%$ of our patients, which is similar to the rate reported by other groups (9.4-17\%) [16, 24]. Clinical manifestations
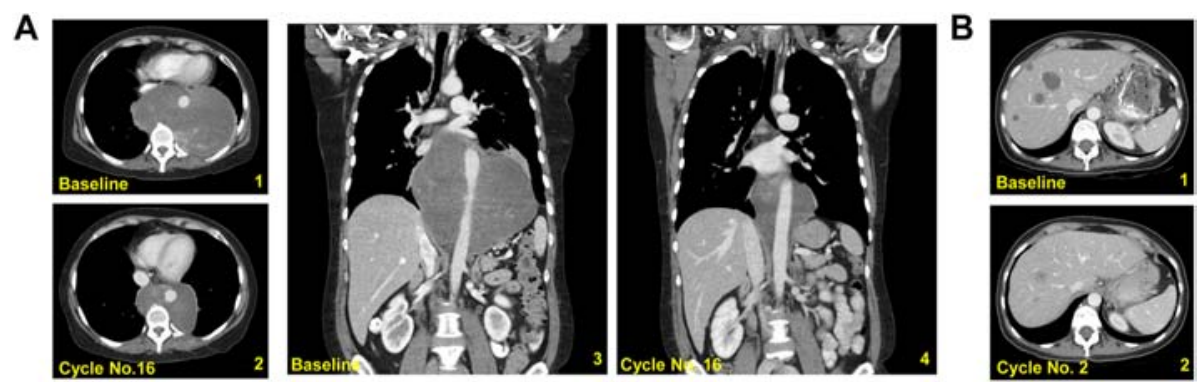

Fig. 4 CT scans of patients who responded to trabectedin. a Patient with recurrent metastatic grade II myxoid liposarcoma encasing the aorta. Paired axial $(1,2)$ and coronal $(3,4)$ cuts showing a partial response after 16 cycles of trabectedin. b Patient with recurrent poorly differentiated leiomyosarcoma that metastasized to the liver. Paired axial $(1,2)$ cuts showing a partial response after 2 cycles of trabectedin 
A

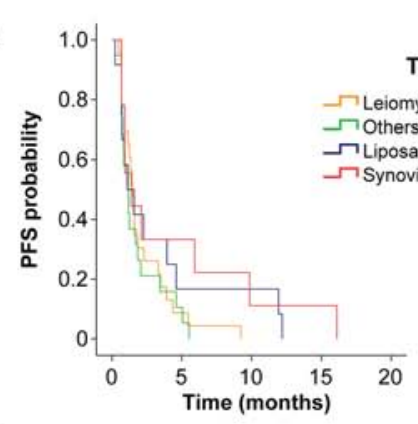

B

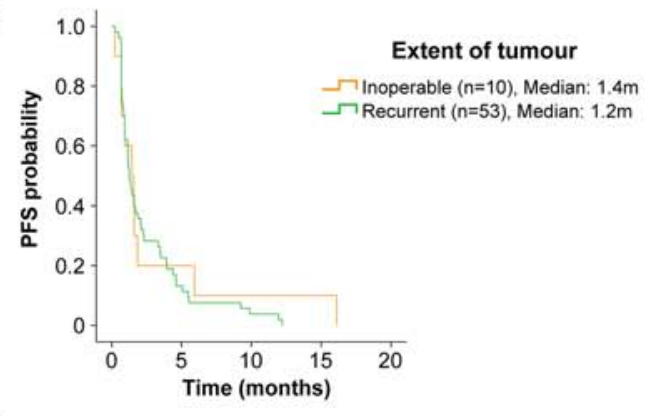

C

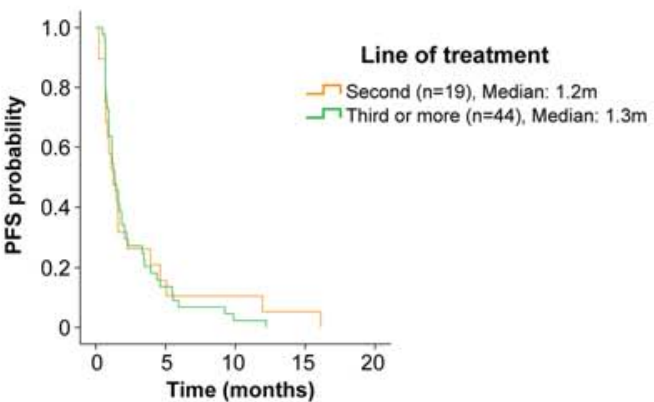

D

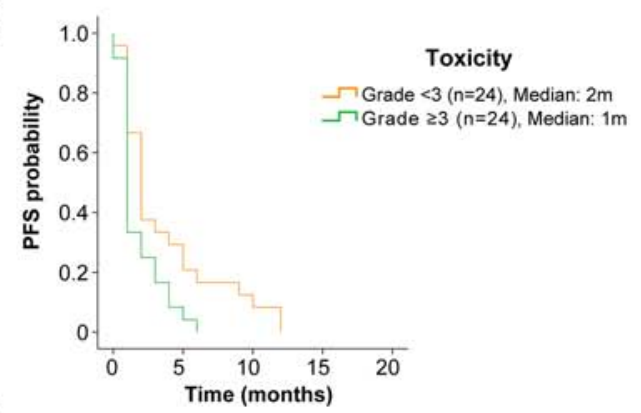

E

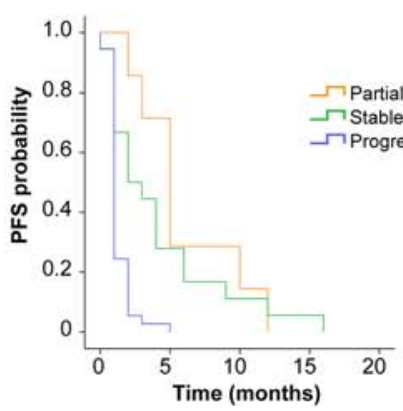

Best response

response $(n=7)$, Median: $5 \mathrm{~m}$ sease ( $n=18)$, Median: $2 m$ disease $(n=37)$, Median: $1 m$
F

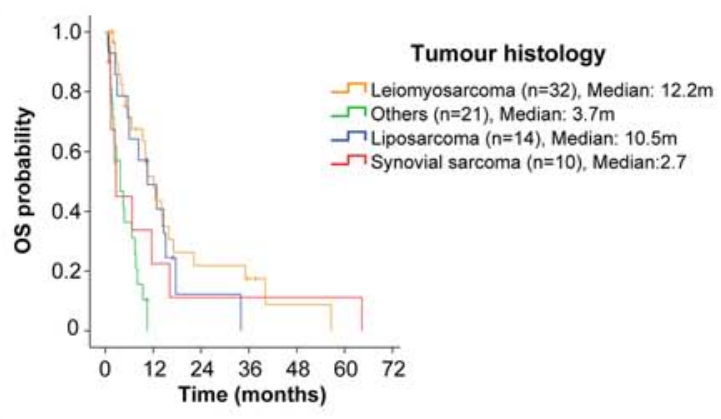

G

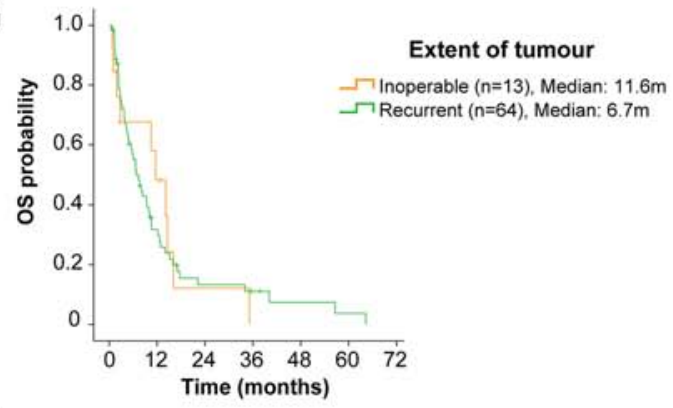

H

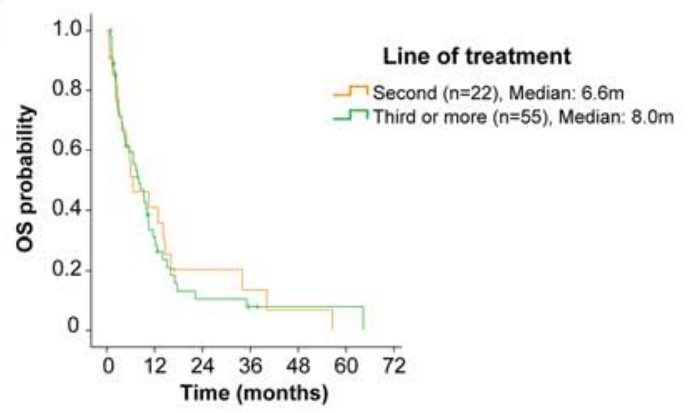

I

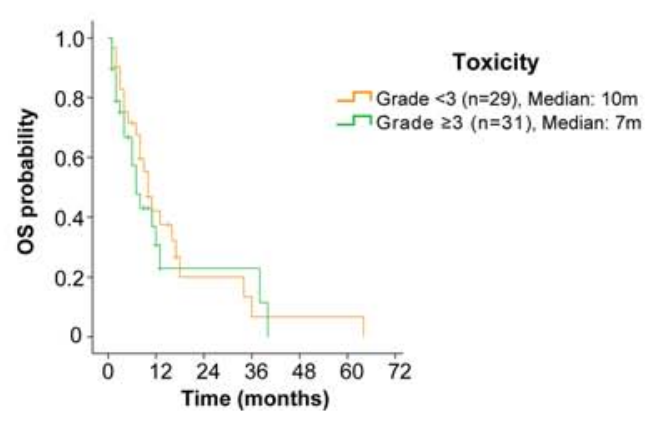

J

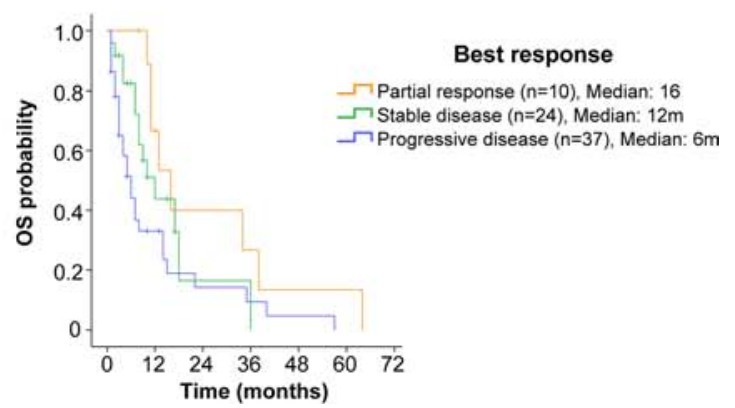

Fig. 5 (See legend on next page.) 
(See figure on previous page.)

Fig. 5 Trabectedin survival analyses. Kaplan-Meier (KM) survival curves for progression-free survival (PFS) (left column) and overall survival (OS) (right column) stratified by tumor histology $(\mathbf{a}, \mathbf{f})$, extent of tumor at presentation $(\mathbf{b}, \mathbf{g})$, line of treatment with trabectedin (c, $\mathbf{h})$, severity of toxicity (d, i) and best response by RECIST (e, j). Abbreviations: m, months; OS, overall survival; PFS, progression free survival, RECIST, Response Evaluation Criteria in Solid Tumors

of severe hepatic injury are rare; therefore the changes observed in liver function tests mainly represent biochemical changes without permanent hepatic injury [25]. Post-treatment liver biopsies showed no evidence of persistent liver histopathological changes attributable to trabectedin [31].

Clinical adverse events were common in our patients. At least $20 \%$ of patients develop clinical symptoms including nausea (64.7\%), fatigue (58.3\%), and vomiting (40.1\%) [25]. Nausea and vomiting, often associated with trabectedin, can be mitigated by pre-treating patients with dexamethasone [32]. Although the mechanism is yet unclear, the protection by dexamethasone against trabectedin-mediated toxicity may be attributed to enhanced Mrp2 biliary excretion and increased metabolism by CYP3A1/2 [33]. Notably, the adverse events commonly induced by cytotoxic chemotherapy or that are potentially dose-limiting, debilitating, cumulative and/ or life threatening are rare with trabectedin [25]. Overall trabectedin's safety profile compares favorably with that of current standard-of-care agents used against STS [34].

Dosage and scheduling adjustments were in concordance with those reported by other studies. Dose reduction was necessary in $19.5 \%$ of patients, which is well within the rate reported in the literature (14-48 \%) [13, $17,24,26]$. As expected the primarily causes for dose reduction were either low ANC or elevated liver enzymes $[13,26]$. Approximately $40 \%$ of our patients required treatment delays. Other studies have reported lower rates (27.7-36 \%), but continue to find that neutropenia and increased transaminases are the two main causes $[13,17,25]$. Treatment discontinuations due to toxicity was necessary in $9.7 \%$ patients, which is similar to the rate reported in the literature $(8-10.2 \%)[17,25]$. The primary reasons for treatment discontinuance include disease progression (63\%) [17].

Deaths attributed to drug-related events occurred in two patients and were attributed to rhabdomyolysis. The reported death rate is $0.5-1.7 \%$ [17, 24, 25]. Deaths generally occur during the first two cycles of treatment and are mainly due to rhabdomyolysis [14, 25, 35]. Less frequently trabectedin causes death by inducing severe myelosuppression and respiratory failure [17]. Periodic monitoring of creatine phosphokinase as well as awareness of clinical manifestations is recommended for timely intervention.
Twenty-three percent of patients in our study received $\geq 6$ cycles. In other studies a slightly higher percentage of patients (30-34\%) received an equivalent number of cycles of treatment suggesting an acceptable toxicity profile that allows prolonged treatment in certain patients $[16,24]$. The number of patients undergoing long-term treatment would have been higher had they not progressed as trabectedin lacks cumulative toxicities [16, 24]. In a study that grouped data from 11 French centres, Blay et al reported that among 56 patients who were not progressing after six cycles, the 40 who continued treatment had a significantly higher PFS and OS relative to other patients [16]. Certainly these results must be taken in context of the retrospective nature of that study nevertheless maintenance therapy in patients with advanced STS is an option worth evaluating.

In our study both the median PFS and OS were on the lower end of what has been previously reported (PFS: $1.7-3.4 \mathrm{~m}$ and OS: $8.9-15.8 \mathrm{~m}$ ) [12, 14, 15, 18, 36]. Our lower survival outcomes may be due to the fact that trabectedin was primarily given as a third- or more line of treatment in the majority of our patients. Other studies have reported higher survival outcomes because patients were not as heavily pre-treated as our cohort. In a study by Le Cesne et al in which only $58.7 \%$ of 885 patients received trabectedin as a third- or more line of treatment, the median PFS and OS were $4.4 \mathrm{~m}$ and $12.2 \mathrm{~m}$, respectively [24]. In another study where $32 \%$ of the cohort received trabectedin as a third or more line of treatment, the median PFS and OS were 3.7 and $8.8 \mathrm{~m}$, respectively [37].

Trabectedin has the potential to provide clinically meaningful benefits to a specific subset of STS patients who have failed standard-of-care treatment particularly if their tumours are either leiomyo- or liposarcoma. Both these subtypes of STS had an OS that was significantly higher relative to patients with other types of STS. This finding was previously shown in other studies in which the median OS was 12-16 m [13, 14, 17]. The severity of toxicities appeared to have an effect on PFS, but not OS as previously shown [16]. A possible explanation is that patients who develop severe toxicities after trabectedin receive a lower number of cycles because trabectedin is discontinued therefore decreasing their chances of responding to treatment. Objective radiological response to treatment as measured by RECIST was also associated with improved OS. Despite the fact that trabectedin 
induced a moderate radiological response (PR and SD) of $48 \%$, as previously reported $[17,36]$, the effect on tumor burden was enough to significantly improve PFS and OS. In another study patients who responded to trabectedin (PR or SD) also had a significantly higher PFS (7.7 $\mathrm{m}$ versus $2.1 \mathrm{~m}, p<0.0001)$ and $\mathrm{OS}(12.1 \mathrm{~m}$ versus $5.5 \mathrm{~m}, p=0.01)$ [37].

\section{Conclusion}

Trabectedin has an acceptable and manageable safety profile and provides encouraging anti-tumor activity particularly in patients with leiomyo- or liposarcoma who failed standard-of-care agents. Trabectedin does not develop cumulative toxicity even in patients who receive a high number of cycles. Response to treatment according to RECIST criteria was modest, especially in patients with lipo- and leiomyosarcoma. OS was significantly improved in patients with leiomyo- or liposarcoma relative to other types of STS. The clinically meaningful benefits provided by trabectedin are comparable to those previously observed in clinical trials and other real-world case series. Our data further support the benefits of trabectedin in patients with advanced leiomyo- and liposarcoma who have failed standard-of-care agents.

\section{Abbreviations \\ ANC: absolute neutrophil count; CR: complete remission; CTCAE: common terminology criteria for adverse events; ECOG: Eastern Cooperative Oncology Group; IQR: interquartile range; M: months; OS: overall survival; PD: progressive disease; PFS: progression-free survival; PR: partial remission; RECSIT: response evaluation criteria in solid tumors; SD: stable disease; STS: soft tissue sarcoma; TTP: time to progression; $Y$ : years old.}

\section{Competing interests}

None declared.

\section{Authors' contribution}

FAA: designed study, collected data, analyzed data, wrote manuscript, edited manuscript. AJC: designed study, collected data, analyzed data, edited manuscript. AR: designed study, analyzed data, edited manuscript. BCD: designed study, analyzed data, edited manuscript. MEB: conceived project, designed study, collected data, analyzed data, edited manuscript. All authors read and approved the final manuscript.

\section{Author details}

${ }^{1}$ Division of General Surgery, Department of Surgery, University of Toronto, Toronto, ON, Canada. ${ }^{2}$ Department of Medical Oncology, Mount Sinai Hospital, Toronto, ON, Canada. ${ }^{3}$ Department of Medical Oncology, Princess Margaret Cancer Centre, University Health Network, Toronto, ON, Canada. ${ }^{4}$ Department of Medicine, University of Toronto, Toronto, ON, Canada. ${ }^{5}$ Department of Pathology and Laboratory Medicine, Mount Sinai Hospital, Toronto, ON, Canada. ${ }^{6}$ Department of Laboratory Medicine and

Pathobiology, University of Toronto, Toronto, ON, Canada.

Received: 30 July 2015 Accepted: 6 January 2016 (t)

\section{References}

1. Surveillance, epidemiology, and end results (SEER) program (www.seer. cancer.gov) SEER*Stat database: Incidence - SEER 9 Regs Research Data, Nov 2010 Sub (1973-2008) < Katrina/Rita population adjustment > Linked to County Attributes - Total U.S., 1969-2009 counties, National Cancer Institute, DCCPS, Surveillance Research Program, Cancer Statistics Branch, Released April 2011, based on the November 2010 submission.
2. National Comprehensive Cancer Network (NCCN). NCCN Clinical Practice Guidelines in Oncology (NCCN Guidelines(R)). Version I. 2015. p. 128.

3. ESMO/European Sarcoma Network Working Group. Soft tissue and visceral sarcomas: ESMO clinical practice guidelines for diagnosis, treatment and follow-up. Ann Oncol. 2014;25 Suppl 3:iii102-12.

4. Van Glabbeke M, van Oosterom AT, Oosterhuis JW, et al. Prognostic factors for the outcome of chemotherapy in advanced soft tissue sarcoma: an analysis of 2,185 patients treated with anthracycline-containing first-line regimens-a European Organization for Research and Treatment of Cancer Soft Tissue and Bone Sarcoma Group Study. J Clin Oncol. 1999:17:150-7.

5. Sleijfer $\mathrm{S}$, Ouali M, van Glabbeke $M$, et al. Prognostic and predictive factors for outcome to first-line ifosfamide-containing chemotherapy for adult patients with advanced soft tissue sarcomas: an exploratory, retrospective analysis on large series from the European Organization for Research and Treatment of Cancer-Soft Tissue and Bone Sarcoma Group (EORTC-STBSG). Eur J Cancer. 2010:46:72-83.

6. Cuevas C, Francesch A. Development of Yondelis (trabectedin, ET-743). A semisynthetic process solves the supply problem. Nat Prod Rep. 2009;26:322-37.

7. D'Incalci M, Galmarini CM. A review of trabectedin (ET-743): a unique mechanism of action. Mol Cancer Ther. 2010;9:2157-63.

8. Allavena P, Signorelli M, Chieppa M, et al. Anti-inflammatory properties of the novel antitumor agent yondelis (trabectedin): inhibition of macrophage differentiation and cytokine production. Cancer Res. 2005;65:2964-71.

9. Germano G, Frapolli R, Simone M, et al. Antitumor and anti-inflammatory effects of trabectedin on human myxoid liposarcoma cells. Cancer Res. 2010;70:2235-44.

10. Germano G, Frapolli R, Belgiovine C, et al. Role of macrophage targeting in the antitumor activity of trabectedin. Cancer Cell. 2013;23:249-62.

11. Forni C, Minuzzo M, Virdis E, et al. Trabectedin (ET-743) promotes differentiation in myxoid liposarcoma tumors. Mol Cancer Ther. 2009;8:449-57.

12. Le Cesne A, Blay JY, Judson I, et al. Phase II study of ET-743 in advanced soft tissue sarcomas: a European Organisation for the Research and Treatment of Cancer (EORTC) soft tissue and bone sarcoma group trial. J Clin Oncol. 2005:23:576-84

13. Garcia-Carbonero R, Supko JG, Manola J, et al. Phase II and pharmacokinetic study of ecteinascidin 743 in patients with progressive sarcomas of soft tissues refractory to chemotherapy. J Clin Oncol. 2004;22:1480-90.

14. Yovine A, Riofrio M, Blay JY, et al. Phase II study of ecteinascidin-743 in advanced pretreated soft tissue sarcoma patients. J Clin Oncol. 2004;22:890-9.

15. Garcia-Carbonero R, Supko JG, Maki RG, et al. Ecteinascidin-743 (ET-743) for chemotherapy-naive patients with advanced soft tissue sarcomas: multicenter phase II and pharmacokinetic study. J Clin Oncol. 2005;23:5484-92.

16. Blay JY, Italiano A, Ray-Coquard I, et al. Long-term outcome and effect of maintenance therapy in patients with advanced sarcoma treated with trabectedin: an analysis of 181 patients of the French ATU compassionate use program. BMC Cancer. 2013;13:64.

17. Samuels BL, Chawla S, Patel S, et al. Clinical outcomes and safety with trabectedin therapy in patients with advanced soft tissue sarcomas following failure of prior chemotherapy: results of a worldwide expanded access program study. Ann Oncol. 2013;24:1703-9.

18. Fayette J, Boyle $\mathrm{H}$, Chabaud S, et al. Efficacy of trabectedin for advanced sarcomas in clinical trials versus compassionate use programs: analysis of 92 patients treated in a single institution. Anticancer Drugs. 2010;21:113-9.

19. Demetri GD, Chawla SP, von Mehren M, et al. Efficacy and safety of trabectedin in patients with advanced or metastatic liposarcoma or leiomyosarcoma after failure of prior anthracyclines and ifosfamide: results of a randomized phase II study of two different schedules. J Clin Oncol. 2009;27:4188-96.

20. Health Canada. Summary basis of decision (SBD) for ${ }^{\text {Pr }}$ Yondelis $^{T M}$. Health Canada. 2010. http://www.hc-sc.gc.ca/dhp-mps/alt_formats/pdf/ prodpharma/sbd-smd/phase1-decision/drug-med/sbd_smd_2010_yondelis_ 124729-eng.pdf. Accessed July 29, 2015.

21. European Medicines Agency. Yondelis: EPAR - product information. European Medicines Agency. 2009. http://www.ema.europa.eu/docs/en_GB/document_ library/EPAR___Product_Information/human/000773/WC500045832.pdf. Accessed July 29, 2015.

22. National Cancer Institute. Common Terminology Criteria for Adverse Events (CTCAE), Version 4.03. National Cancer Institute. 2010. http://evs.nci.nih.gov/ ftp1/CTCAE/CTCAE 4.03_2010-06-14_QuickReference_5x7.pdf. Accessed July 29, 2015. 
23. Eisenhauer EA, Therasse P, Bogaerts J, et al. New response evaluation criteria in solid tumours: revised RECIST guideline (version 1.1). Eur J Cancer. 2009; 45:228-47.

24. Le Cesne A, Ray-Coquard I, Duffaud F, et al. Trabectedin in patients with advanced soft tissue sarcoma: A retrospective national analysis of the French Sarcoma Group. Eur J Cancer. 2015;51:742-50.

25. Le Cesne A, Yovine A, Blay JY, et al. A retrospective pooled analysis of trabectedin safety in 1,132 patients with solid tumors treated in phase II clinical trials. Invest New Drugs. 2012;30:1193-202.

26. Gounaris I, Hatcher HM, Davidson D, et al. Trabectedin for advanced soft tissue sarcomas: a single institution experience. Future Oncol. 2014;10:1843-51.

27. Hing J, Perez-Ruixo JJ, Stuyckens K, et al. Mechanism-based pharmacokinetic/ pharmacodynamic meta-analysis of trabectedin (ET-743, Yondelis) induced neutropenia. Clin Pharmacol Ther. 2008;83:130-43.

28. Cassier PA, Dufresne A, Blay JY, et al. Trabectedin and its potential in the treatment of soft tissue sarcoma. Ther Clin Risk Manag. 2008;4:109-16.

29. Huygh G, Clement PM, Dumez H, et al. Ecteinascidin-743: evidence of activity in advanced, pretreated soft tissue and bone sarcoma patients. Sarcoma. 2006;2006:56282.

30. Delaloge S, Yovine A, Taamma A, et al. Ecteinascidin-743: a marine-derived compound in advanced, pretreated sarcoma patients-preliminary evidence of activity. J Clin Oncol. 2001;19:1248-55.

31. Yver A, Cohen R, Williams D, et al. Assessment of trabectedin (T) induced liver toxicity with correlation to liver morphology in a phase I study of T+ pegylated liposomal doxorubicin (PLD). J Clin Oncol. 2006;24:536s.

32. Taamma A, Misset $J \mathrm{~L}$, Riofrio $\mathrm{M}$, et al. Phase I and pharmacokinetic study of ecteinascidin-743, a new marine compound, administered as a 24-hour continuous infusion in patients with solid tumors. J Clin Oncol. 2001;19:1256-65.

33. Lee JK, Leslie EM, Zamek-Gliszczynski MJ, et al. Modulation of trabectedin (ET-743) hepatobiliary disposition by multidrug resistance-associated proteins (Mrps) may prevent hepatotoxicity. Toxicol Appl Pharmacol. 2008; 228:17-23.

34. van Oosterom AT, Mouridsen HT, Nielsen OS, et al. Results of randomised studies of the EORTC Soft Tissue and Bone Sarcoma Group (STBSG) with two different ifosfamide regimens in first- and second-line chemotherapy in advanced soft tissue sarcoma patients. Eur J Cancer. 2002:38:2397-406.

35. McMeekin DS, Lisyanskaya A, Crispens M, et al. Single-agent trabectedin as second-line therapy of persistent or recurrent endometrial cancer: results of a multicenter phase II study. Gynecol Oncol. 2009;114:288-92.

36. Roylance R, Seddon B, McTiernan A, et al. Experience of the use of trabectedin (ET-743, Yondelis) in 21 patients with pre-treated advanced sarcoma from a single centre. Clin Oncol (R Coll Radiol). 2007;19:572-6.

37. Schmitt T, Keller E, Dietrich $\mathrm{S}$, et al. Trabectedin for metastatic soft tissue sarcoma: a retrospective single center analysis. Mar Drugs. 2010;8:2647-58.

\section{Submit your next manuscript to BioMed Central and we will help you at every step:}

- We accept pre-submission inquiries

- Our selector tool helps you to find the most relevant journal

- We provide round the clock customer support

- Convenient online submission

- Thorough peer review

- Inclusion in PubMed and all major indexing services

- Maximum visibility for your research

Submit your manuscript at www.biomedcentral.com/submit

) Biomed Central 